\title{
Structural valve deterioration of bioprosthetic aortic valves: An underestimated complication
}

\author{
Thomas Sénage, MD, PhD, ${ }^{\mathrm{a}, \mathrm{b}}$ Florence Gillaizeau, $\mathrm{PhD}$, ${ }^{\mathrm{a}, \mathrm{d}, \mathrm{d}}$ Thierry Le Tourneau, MD, PhD, ${ }^{\mathrm{e}, \mathrm{f}}$ \\ Basile Marie, MD, ${ }^{\mathrm{b}}$ Jean-Christian Roussel, MD, PhD, ${ }^{\mathrm{b}}$ and Yohann Foucher, $\mathrm{PhD}^{\mathrm{a}, \mathrm{c}}$
}

\section{ABSTRACT}

Objectives: Structural valve deterioration (SVD) remains a major bioprosthesisrelated complication, as recently described for the Mitroflow valve (models LX and 12A) (LivaNova, London, United Kingdom). The real incidence of the SVD risk remains unclear, often due to methodologic pitfalls by systematically using the Kaplan-Meier estimator and/or the Cox model. In this report, we propose for the first time a precise statistical modeling of this issue.

Methods: Five hundred sixty-one patients who underwent aortic valve replacement with the aortic Mitroflow valve between 2002 and 2007 were included. We used an illness-death model for interval-censored data. Median follow-up was 6.6 years; 103 cases of SVD were diagnosed.

Results: The 4-year and 7-year SVD cumulative incidences after the first anniversary of surgery were $15.2 \%$ (95\% confidence interval, 11.9-19.1) and $31.0 \%$ (95\% confidence interval, 25.8-37.2), respectively. Female gender, dyslipidemia, chronic obstructive pulmonary disease, and severe patientprosthesis mismatch were significant risk factors of SVD. The occurrence of SVD was associated with a 2 -fold increase in the risk of death.

Conclusions: Appropriate statistical models should be used to avoid underestimating the SVD complication associated with worse long-term survival. (J Thorac Cardiovasc Surg 2019;157:1383-90)

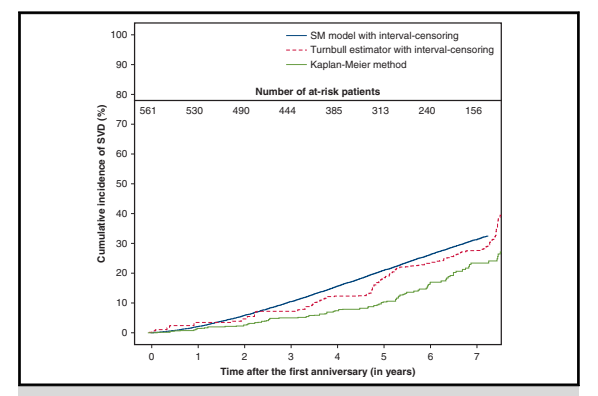

The cumulative incidence of SVD: Misestimated by inappropriate methodology.

\section{Central Message}

Appropriate statistical models should be used so the SVD complication is not underestimated.

\section{Perspective}

The SVD complication may have been underestimated for some bioprostheses due to methodologic pitfalls. Patient death should be considered as a competing event for a proper estimation of the cumulative incidence. Furthermore, the possible interval censoring of such cardiovascular events has also to be considered. We propose for the first time a precise statistical modeling of this issue.

See Commentary on page 1391.
More than 200,000 aortic valve replacement (AVR) procedures are performed yearly worldwide. ${ }^{1}$ Due to enhanced tissue durability, the choice of prosthesis has changed in favor of biological prostheses. Structural valve deterioration (SVD) remains a major bioprosthesis-related

\footnotetext{
From ${ }^{\mathrm{a}}$ INSERM UMR 1246 - SPHERE for Nantes University, Tours University, France, ${ }^{b}$ Cardiothoracic Surgical Unit, Thorax Institute, ${ }^{\mathrm{c} C e n t e r}$ for Research in Transplantation and Immunology, Institute of Transplantation Urology and Nephrology, ${ }^{e}$ Department of Physiology, Thorax Institute, ${ }^{f}$ National Center for Scientific Research (CNRS) UMR 6291, for University of Nantes, St Herblain, France, and ${ }^{\mathrm{d}}$ Department of Statistical Science for University College London, London, United Kingdom.

Received for publication June 14, 2017; revisions received July 30, 2018; accepted for publication Aug 3, 2018; available ahead of print Nov 8, 2018.

Address for reprints: Thomas Sénage, MD, PhD, Cardiac Surgical Unit, Thorax Institute, University Hospital of Nantes, Bd Monod, 44035 St Herblain, France (E-mail: thomas.senage@chu-nantes.fr).

$0022-5223 / \$ 36.00$

Copyright (C) 2018 by The American Association for Thoracic Surgery https://doi.org/10.1016/j.jtcvs.2018.08.086
}

complication, with significant heterogeneity among the types of prosthesis. ${ }^{2,3}$ Among bovine pericardial prostheses, the Mitroflow valve (LivaNova, London, United Kingdom), available since 1982, was designed to improve prosthesis hemodynamic performance, especially in small aortic roots thanks to its lower bulk. ${ }^{4}$ More than 100,000 AVR procedures have been performed with this valve worldwide since its first implantation.

It is reasonable to assume that SVD incidence is currently underestimated. The main reason for this is the macroscopic

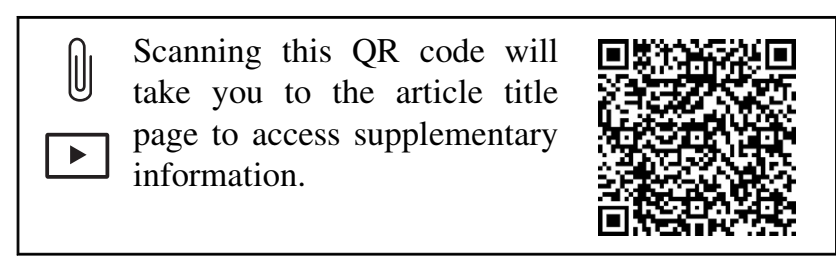




\section{Abbreviations and Acronyms \\ AVR = aortic valve replacement \\ $\mathrm{COPD}=$ chronic obstructive pulmonary disease \\ $\mathrm{EAO}=$ effective orifice area \\ PPM = patient-prosthesis mismatch \\ SVD = structural valve deterioration \\ TAVI $=$ transcatheter aortic valve implantation}

or histologic definition of SVD often used in the literature, ${ }^{5,6}$ whereas high-risk octogenarian patients are referred to surgery less frequently following SVD diagnosis. ${ }^{7,8} \mathrm{In}$ agreement with recent recommendations, ${ }^{9}$ we therefore studied the SVD risk based on echocardiographic criteria in a cohort of patients who underwent AVR with the Mitroflow bioprosthesis (12A and LX models). ${ }^{10}$ By using the usual Kaplan-Meier estimator, we estimated the 5-year cumulative incidence of diagnosed SVD at $8.4 \%(95 \%$ confidence interval $[\mathrm{CI}], 5.3-11.3)$. Moreover, using a Cox model with time-dependent covariates, we estimated a 7.7-fold higher risk of death after SVD diagnosis $(95 \%$ CI, 4.4-13.6). Our study was among the first to report this health issue.

We believe that the true incidence of SVD is still underestimated. Firstly, the time-to-SVD was intervalcensored. There are 2 types of incomplete data: for patients with SVD, the time-to-SVD between the last normal echocardiogram and the first abnormal echocardiogram is unknown; and for patients who died without SVD diagnosis, the SVD may have occurred after their last normal echocardiogram. Ignoring this interval-censored process by considering death as right-censoring may also result in an overestimated mean time-to-SVD and an incorrect estimation of the relationship between SVD incidence and the risk of death. ${ }^{11}$ Secondly, the median follow-up time was 4.1 years, whereas SVD is a long-term event.

The aim of the present study was to more accurately estimate the incidence of SVD for patients who underwent an AVR with a Mitroflow bioprosthesis, along with its influence on patient mortality. For that purpose, we updated the patients' follow-up data from our previous study ${ }^{10}$ to better appraise long-term outcomes. We also developed an original statistical method to deal with interval censoring; that is, a semi-Markov illness-death model.

\section{MATERIAL AND METHODS \\ Patients}

Between January 2002 and December 2007, 617 consecutive patients underwent AVR with a Mitroflow bioprosthesis (12A and LX models) in the Nantes University Hospital, St Herblain, France. The valve was prepared preoperatively as indicated in the user manual. ${ }^{12}$ SVD is unlikely to occur during within the first year postsurgery; we observed no event during this period. To study the incidence of SVD and its influence on mortality, the baseline of our study was defined as the first anniversary of the surgery. Five hundred sixty-one patients alive without SVD at 1-year postsurgery were included. More precisely, the 26 patients who died in hospital and the 30 patients discharged from hospital but who died before the first anniversary of surgery were not included. In case of redo-surgery or transcatheter aortic valve implantation (TAVI) occurring during the follow-up, with or without diagnosis of SVD, the data were censored at the time of the procedure.

\section{Data Collection}

Perioperative data were collected prospectively, whereas echocardiograms and vital signs were retrospectively collected. The long-term follow-up of the patients included in this cohort was ensured by the patients' own cardiologists. Clinical and echocardiographic data were collected by the Clinical Investigation Centre at the Nantes University Hospital after authorization by the local ethics committee (ie, the institutional review board) and the Commission Nationale Informatique Libertés (authorizations 1456630v1 and 910300). Morbidity and mortality were analyzed taking into account the recommendations of the American Association for Thoracic Surgery, the Society of Thoracic Surgery, and European Association for Cardio-Thoracic Surgery. ${ }^{9}$

The following preoperative data were collected: age; sex; body mass index; family history; high blood pressure history; diabetes mellitus; dyslipidemia; obesity; history of smoking; aortic valve disease (ie, stenosis, insufficiency, mixed disease, endocarditis, or prosthetic endocarditis); New York Heart Association functional classification; pulmonary edema; syncope; atrial fibrillation; chronic obstructive pulmonary disease (COPD); second forced expiratory volume; peripheral vascular disease; renal failure (creatinine $>200 \mu \mathrm{mol} / \mathrm{L}$ or Cockcroft-Gault creatinine clearance $<60 \mathrm{~mL} / \mathrm{min}$ ); preoperative dialysis; stroke; carotid or coronary stenosis; myocardial infarction; left ventricular ejection fraction; systolic pulmonary arterial pressure $>60 \mathrm{~mm} \mathrm{Hg}$; and elective, urgent, or emergency procedure. Severe prosthesis-patient mismatch (PPM) was defined as an effective orifice index area of the aortic prosthesis $\leq 0.65 \mathrm{~cm}^{2} / \mathrm{m}^{2},{ }^{13}$ based on the in vivo effective orifice area (EOA) given by the manufacturer. ${ }^{12}$

The following explicative variables were studied: Severe PPM, body mass index, age at time of surgery, sex, dyslipidemia, COPD, diabetes mellitus, left ventricular ejection fraction, atrial fibrillation, peripheral vascular disease, and isolated AVR.

\section{SVD Definition}

The SVD definition we used was defined based on several international recommendations. ${ }^{14}$ Indeed, until very recently, no standardized definition was available. We therefore used in this study an ad hoc definition: progression of aortic transprosthetic gradient $\geq 30 \mathrm{~mm} \mathrm{Hg}$ associated with a decrease in EOA $\leq 1 \mathrm{~cm}^{2}$ and aortic cusp alteration, or intraprosthetic aortic regurgitation $>2$ out of 4 . Long-term patient follow-up was performed by these latters' own personal cardiologists (outside our institution where the surgeries where performed). The echocardiography times were not planned for our study, we only observed real-life practices. Each case of SVD was carefully assessed and validated following a review of medical reports. In cases where SVD was suspected on echocardiography but not ascertained, patients were referred to our institution.

\section{Statistical Analysis}

The median follow-up time was estimated using the reverse Kaplan-Meier method. ${ }^{15}$ We used a semi-Markov illness-death model (Appendix 1). This model allowed us to study the SVD as an intervalcensored event before death. ${ }^{16,17}$ This interval censoring is important when dealing with an event that is known to occur within an interval instead of being observed exactly. For our cohort, the event date for patients with SVD was known to have occurred between the last normal echocardiogram and the diagnostic echocardiogram. Similarly, patients 
who died without SVD diagnosis may have developed the SVD after their last normal echocardiogram. The illness-death model we propose aims to estimate the probability of SVD before death. Indeed, our aim was to estimate the properties of the valve in a real-life setting: one can accept that a valve may deteriorate, but SVD must occur after the patient's death. Compared with nonparametric approaches, this approach presents the advantage of requiring fewer parameters with a smaller sample size, estimating an adjusted hazard ratio (HR) due to covariates, and allowing us to model the distribution of the time to transition from the illness to death state.

Patients who underwent repeat AVR unrelated to SVD were right-censored at the time of the new surgery $(n=7)$. The generalized Weibull distribution was tested for the 3 transition-specific baseline hazard functions, and the model was secondarily simplified without unnecessary parameters (an exponential distribution was chosen for the transition from SVD to death). The assumption of proportional hazards was graphically assessed. Risk factors were initially selected from univariable models (Wald test $P<.25$ ). A multivariable model was then estimated with a backward procedure performed manually, variable by variable, for each transition (Wald test $P<.05$ ). The illness-death model was used to estimate: the cumulative incidence of SVD and the HR of death related to SVD incidence by combining bootstrapping and simulations (for each bootstrap sample, the times to event were simulated according to the final multivariable model and the observed baseline characteristics of the patients and the HR was estimated from a time-dependent Cox model. ${ }^{18}$

The description of the semi-Markov model is presented in the supplemental material. The nonparametric Turnbull estimator for interval-censored data and the Kaplan-Meier method were used to estimate the cumulative incidence of SVD and compared with the results from the illness-death model (Figure 1).

To illustrate the need for appropriate methods, we completed the evaluation of our model regarding the competing risk analysis and the interval censoring with comparisons of results obtained by different methods. We compared the Cumulative Incidence Functions (CIFs) of SVD obtained by the proposed semi-Markov illness-death model, the same model without taking into account interval-censoring, the Aalen and Johansen estimator ${ }^{19}$ (Figure E2), the nonparametric Turnbull estimator for interval-censored data, and the Kaplan-Meier method (Figure 1). To obtain data with no interval censoring, we considered the time-to-SVD as the mean between the lower and the upper bounds of the interval. Patients without SVD diagnosis were considered free of SVD at the end of the follow-up.

Time-to-SVD was defined in the middle of the interval between the normal and abnormal echocardiograph reports. Patients who died with no SVD diagnosis were right-censored at the date of death.

Statistical analyses were performed with $\mathrm{R}$ software version 3.1.1 (R Foundation for Statistical Computing, Vienna, Austria), along with the multistate ${ }^{20}$ and relsurv packages. ${ }^{21}$

\section{RESULTS \\ Baseline Cohort Characteristics}

The preoperative characteristics of the 561 patients are presented in Table 1. The mean age was $76.4 \pm 6.0$ years. Fifty-seven percent of patients $(\mathrm{n}=321)$ were aged between ages 70 and 80 years and $29 \%(n=162)$ were octogenarians. Women represented $56 \%(\mathrm{n}=314)$ of the patients. The indication for surgery was aortic valve stenosis in $83.6 \%$ of patients $(\mathrm{n}=469)$. The proportion of repeat surgery was $5.5 \%(\mathrm{n}=31)$. Isolated AVR was performed in 362 patients $(64.5 \%)$. The associated

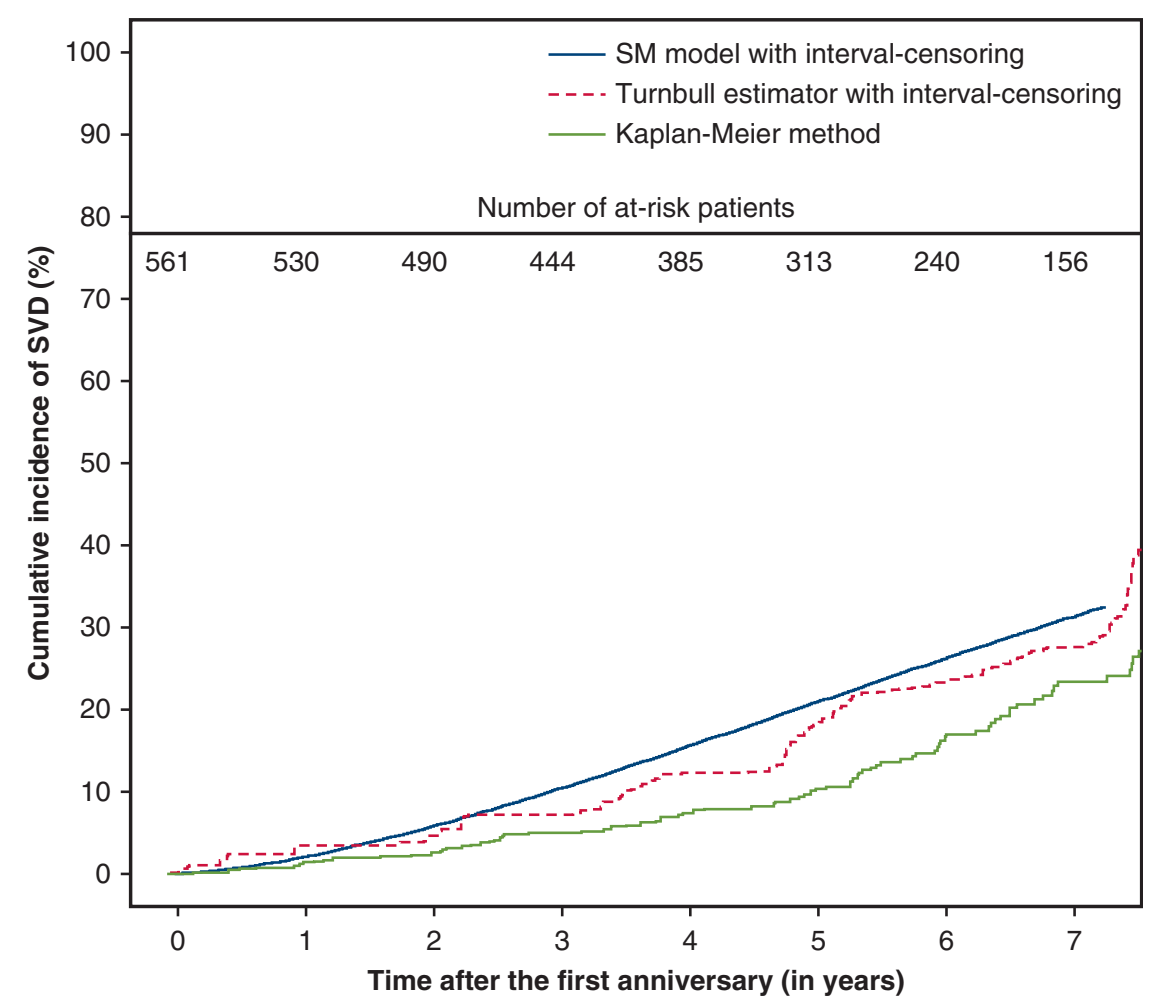

FIGURE 1. Cumulative incidence of structural valve deterioration (SVD) after the first anniversary of surgery. The blue curve was estimated from the illness-death model handling interval censoring for time-to-SVD and competition with death without SVD. The green curve was estimated with the Kaplan-Meier method. The red line was estimated from Turnbull's non-parametric estimator for interval censored data. SM, Semi-Markov. 
TABLE 1. Baseline characteristics of the monocentric cohort comprising 561 patients who underwent an aortic valve replacement (AVR) with a Sorin Mitroflow bioprosthesis (LivaNova, London, United Kingdom)

\begin{tabular}{|c|c|}
\hline Clinical data & Result \\
\hline Female sex & $314(56.0)$ \\
\hline Age $(y)$ & $76.4 \pm 6.0$ \\
\hline Body mass index & $26.7 \pm 4.8$ \\
\hline Atrial fibrillation & $86(15.3)$ \\
\hline High blood pressure & $352(62.8)$ \\
\hline Mellitus diabetes & $111(19.8)$ \\
\hline Dyslipidemia & $257(45.8)$ \\
\hline Obesity & $129(23.0)$ \\
\hline History of smoking & $91(16.2)$ \\
\hline NYHA functional classification III or IV & $167(29.8)$ \\
\hline \multicolumn{2}{|l|}{ Comorbidities } \\
\hline Peripheral vascular disease & $71(12.7)$ \\
\hline Preoperative renal failure & $37(6.6)$ \\
\hline Chronic obstructive pulmonary disease & $33(5.9)$ \\
\hline Stroke & $18(3.2)$ \\
\hline Myocardial infarction history & $30(5.3)$ \\
\hline Coronary angioplasty & $29(5.2)$ \\
\hline \multicolumn{2}{|l|}{ Echocardiography } \\
\hline Left ventricular ejection fraction, percentage & $58 \pm 12$ \\
\hline Left ventricular ejection fraction $<50 \%$ & $68(12.1)$ \\
\hline Systolic pulmonary artery pressure $>60 \mathrm{~mm} \mathrm{Hg}$ & $16(2.9)$ \\
\hline Aortic stenosis & $469(83.6)$ \\
\hline Aortic insufficiency & $16(2.6)$ \\
\hline \multicolumn{2}{|l|}{ Surgical data } \\
\hline Elective surgery & $486(86.6)$ \\
\hline Implanted bioprostheses $\leq 21 \mathrm{~mm}$ & $364(64.9)$ \\
\hline Severe PPM & $131(23.4)$ \\
\hline Logistic EuroSCORE & $10.0 \pm 10.1$ \\
\hline
\end{tabular}

Values are presented as $\mathrm{n}(\%)$ or mean \pm standard deviation. NYHA, New York Heart Association; PPM, patient-prosthesis mismatch; EuroSCORE, European system for cardiac operative risk evaluation.

procedures were mainly coronary artery bypass surgery in $30.3 \%$ of patients $(\mathrm{n}=170)$. A small diameter prosthesis (19 or $21 \mathrm{~mm})$ was implanted in $64.9 \%(\mathrm{n}=364)$ of patients and $23.4 \%$ of patients $(n=131)$ had severe PPM.

\section{Description of the Follow-up}

The median follow-up time was 6.6 years (range, 0-10.6 years) after the first anniversary of surgery. Two thousand one hundred sixty-four echocardiograms were collected. The median number of echocardiogram reports by patient was 3 , with a maximum of 10 . Nine patients were included without echocardiogram assessment. The maximum follow-up was 10.6 years after the first anniversary of surgery, with a mean interval between 2 follow-ups of 458 days (min-max, 5-3280 days). SVD was diagnosed in 103 patients. More precisely, 298 patients were still alive without SVD diagnosis, 160 patients died without previous SVD diagnosis, 73 patients were still alive after SVD diagnosis, and 30 patients died following SVD diagnosis. Two SVD modes were observed: the main was calcified prosthetic stenosis (Figure 2) in 81 patients, whereas moderate to severe intraprosthetic regurgitation was found in 22 patients. Among patients diagnosed with SVD, $35.0 \%(\mathrm{n}=36)$ were not referred by personal cardiologists, $38.8 \%(\mathrm{n}=40)$ underwent a repeat AVR $(\mathrm{n}=31$, Video 1$)$ or TAVI $(\mathrm{n}=9), 15.5 \%(\mathrm{n}=16)$ were denied surgery and TAVI after clinical workup, and 3.9\% $(\mathrm{n}=4)$ refused the clinical workup. Four patients $(3.9 \%)$ died while waiting for repeat surgery. Among the 190 deaths, SVD was among the main reported causes of death in $10.0 \%$ of cases $(n=19)$. The other reported causes (Table E1) were congestive heart failure $(17.9 \%$ $[\mathrm{n}=34])$, cancer $(12.6 \% \quad[\mathrm{n}=24])$, and sepsis $(9.5 \%[\mathrm{n}=18])$. The overall survival compared with the valve-related and cardiac-related survival of this cohort is illustrated in Figure E3.

\section{Cumulative Incidence of SVD}

The first SVD was diagnosed 2 months after the first year of surgery (14 months after surgery). As illustrated by Figure 1, the 4- and 7-year cumulative incidences after the first anniversary of surgery were, respectively, $7.2 \%$ (95\% CI, 4.8-9.5) and 23.4\% (95\% CI, 18.3-28.2) for the Kaplan-Meier estimator, $11.9 \%$ (95\% CI, 8.3-15.5) and $28.3 \% \quad(95 \%$ CI, 22.6-36.4) for the Turnbull estimators, and 15.2\% (95\% CI, 11.9-19.1) and 31.0\% (95\% CI, 25.8-37.1) for the semi-Markov model. As expected, by comparing the results obtained using the Kaplan-Meier and Turnbull estimators, ignorance of the interval-censored process leads to an underestimation of the cumulative incidence of SVD. Moreover, by comparing the results obtained by our semi-Markov illness-death model and the Turnbull estimator, one can conclude that ignorance of competing risks could still lead to an underestimation of the midterm cumulative incidence of SVD.

As listed in Table 2, the illness-death model indicated that the significant risk factors of SVD were female sex (HR, 1.6; 95\% CI, 1.1-2.4), dyslipidemia (HR, 1.6; 95\% CI, 1.1-2.3), severe PPM (HR, 1.7; 95\% CI, 1.1-2.5), and COPD (HR, 2.9; 95\% CI, 1.6-5.4). According to this model, we estimated a 2-fold increased risk of death (95\% CI, 1.3-2.9) after SVD incidence.

\section{DISCUSSION}

We aimed to accurately estimate the incidence of SVD and its relationship with the risk of death in patients who underwent AVR with the Mitroflow valve models 12A and LX. In our previous study, ${ }^{10}$ the cumulative incidence of SVD reached 8.4\% (Kaplan-Meier estimator, 95\% CI, 


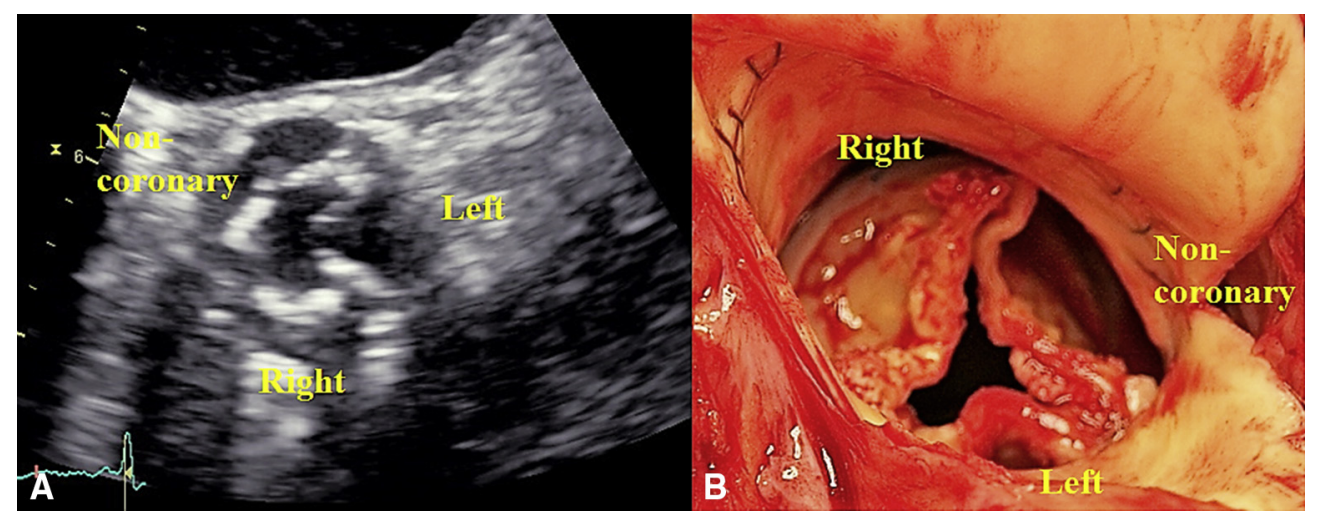

FIGURE 2. Mitroflow bioprosthesis valve $(21 \mathrm{~mm}$ ) (LivaNova, London, United Kingdom) structural failure characterized by commissural calcifications and global thickening of the 3 cusps just before reoperation. A, Transesophageal echocardiography showing thickening and calcification of the bioprosthesis. B, Mitroflow valve during explantation showing calcified nodules, particularly in the commissural regions, and diffuse cusp infiltration. Left, Left cusp; Right, right cusp.

5.3-11.3) at 5 years postsurgery. But the usual methods we used failed to handle several specific issues relating to the SVD risk assessment. As recently pointed out by Huebner and colleagues, ${ }^{22}$ methodologic pitfalls are frequent in the literature, even in prominent medical journals, leading to biased estimations. In our study, we developed an illnessdeath model, a statistical approach that deals with the competing risk and interval censoring of SVD. The 5-year postsurgery cumulative incidence of SVD was estimated at $15.2 \%(95 \% \mathrm{CI}, 11.9-19.1)$, underlining a possible underestimation of the incidence of SVD in our previous study. Thanks to an extended follow-up, we also described a cumulative incidence of SVD of $31.0 \%(95 \%$ CI, 25.8-37.2) at 7 years after the first anniversary of the surgery.

Additionally, we highlighted for the first time (as far as we know) that COPD constitutes a significant risk factor for SVD. Its prevalence was $5.9 \%$ in our cohort. The mechanism seems unclear, but inhalation exposures, as in COPD, can lead to local and general inflammatory

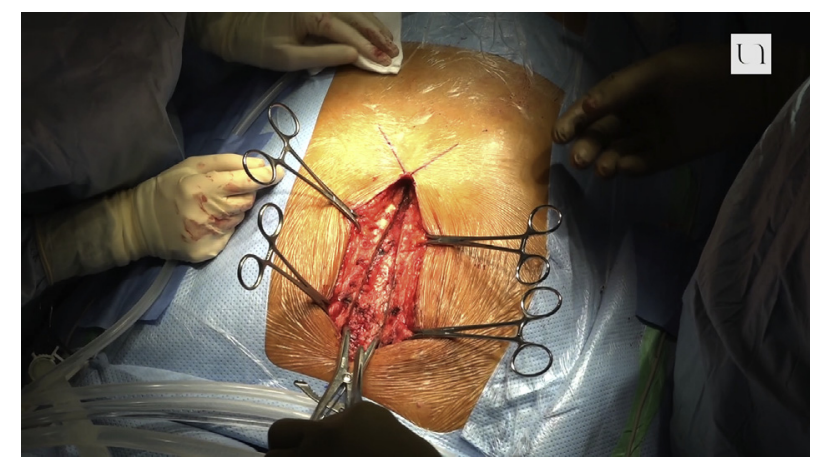

VIDEO 1. Redo aortic valve replacement (AVR) for structural valve deterioration. (Male patient, aged 53 years, first implantation in 2011, redo-AVR in November 2017). Video available at: https://www.jtcvs.org/ article/S0022-5223(18)32480-2/fulltext. responses, which increase with disease severity. Moreover, respiratory infections are more frequent in patients with COPD and can also increase the systemic inflammatory response. Thus, repetitive inflammatory responses could increase the immune response, which has been reported to potentially increase the SVD process. ${ }^{23}$ This hypothesis needs to be confirmed, but it could pave the way for preventing SVD.

Other risk factors were significantly linked to SVD incidence. In accordance with the literature, we identified dyslipidemia $^{24,25}$ and PPM. ${ }^{26,27}$ Flameng and colleagues ${ }^{26}$ assumed that the role of PPM could be due to disturbed flow patterns. Annulus enlargement is thus currently recommended by some authors in patients with predictable severe PPM, ${ }^{28}$ but with an increased risk of procedurerelated surgical complications. ${ }^{29,30}$ The prevalence of PPM in our study was $23.4 \%$, a proportion quite different from the literature. For example, Jamieson and colleagues ${ }^{31}$ reported only $2.9 \%$ of severe PPM with 19-mm Mitroflow valve and $0.5 \%$ overall. However, the in vivo EOA was validated from a small number of patients and was clearly greater than in the prespecified information provided by the company; for instance, an in vivo EOA of $1.4 \mathrm{~cm}^{2}$ for the $19-\mathrm{mm}$ Mitroflow valve versus $1.1 \mathrm{~cm}^{2}$ $\left(1.1 \mathrm{~cm}^{2}\right.$ was the in-vivo EOA for the $19-\mathrm{mm}$ Mitroflow; $1.4 \mathrm{~cm}^{2}$ was the EAO provided by the company. The same difference was present for the 21-mm valve.). ${ }^{12}$ With similar values, the severe PPM rates in our cohort would have been $0 \%$ and $2.4 \%$ for the $19-$ and $21-\mathrm{mm}$ dimensions, respectively.

In our previous study based on a Cox model, ${ }^{10}$ SVD diagnosis was associated with a significant 7.7-fold increase in the risk of death (95\% CI, 4.4-13.6). We additionally proposed a precise appraisal of the influence of SVD on patient survival. Based on the illness-death model, we estimated in the present study a 2-fold higher risk of death after SVD occurrence (95\% CI, 1.3-2.9). These results are 
TABLE 2. Significant factors associated with the time-to-structural valve defect after the first anniversary of surgery in the illness-death model (forced age adjustment; $\mathbf{n}=\mathbf{5 6 1}$ )

\begin{tabular}{lcc}
\hline \multicolumn{1}{c}{ Factor } & Hazard ratio $(\mathbf{9 5} \%$ Confidence interval) & $\boldsymbol{P}$ value \\
\hline Severe patient-prosthesis mismatch & $1.68(1.13-2.49)$ & .010 \\
Female sex & $1.59(1.06-2.38)$ & .026 \\
Dyslipidemia & $1.56(1.07-2.27)$ & .019 \\
Chronic obstructive pulmonary disease & $2.91(1.56-5.44)$ & .001 \\
Younger than age $70 \mathrm{y}$ at the time of surgery & 1.00 & .867 \\
$\quad$ Between ages 70 and 80 y at the time of surgery & $1.05(0.62-1.78)$ & .751 \\
\hline Older than age $80 \mathrm{y}$ at the time of surgery & $0.90(0.47-1.72)$ & \\
\hline
\end{tabular}

in stark contrast with the studies that described a very low percentage of SVD with the Mitroflow valve. 5,6,32,33 These differences are likely related to the methodologic pitfalls we described. We therefore hope that the methodologic framework we propose in this study; that is, the definition of SVD based on echocardiograms and the illness-death model for interval-censored data and competing events, will be further considered in future AVR-related studies. But as is always the case for observational studies of this kind, several limitations must be underlined. Firstly, we deliberately limited the study to patients alive 1 year postsurgery, due to the absence of observed SVD within this interval. We definitely believe that deterioration is a continuous process, with a minimum duration necessary to achieve a progression of aortic transprosthetic gradient $\geq 30 \mathrm{~mm} \mathrm{Hg}$ associated with a decrease in EOA $\leq 1 \mathrm{~cm}^{2}$ and aortic cusp alteration, or intraprosthetic aortic regurgitation $>2$ out of 4 . Nevertheless, this first period may not be strictly equal to 1 year and may vary according to the cohort, the studied valve(s), and the SVD definition. Secondly, although we updated the follow-up of this cohort, the follow-up period still does not allow a precise estimation of long-term SVD cumulative incidence (the curve is still increasing at this maximum time in Figure 2). Thirdly, the echocardiograms were not planned, but were implemented by personal cardiologists, as in real medical practice. We would have preferred scheduled echocardiograms with identical intervals between 2 follow-up visits. In our real-life study, variable times between 2 clinical examinations can be observed. One can suppose a dependence between the follow-ups and the health state of the patients, representing a possible information bias in our results. For instance, a patient presenting with increased dyspnea or cardiac symptoms would be more likely to visit his/her cardiologist. Consequently, the incidence of SVD may still be underestimated by omitting asymptomatic SVD. However, even for studies with the benefit of regular echocardiograms (for instance every 2 years), interval-censoring data and competing events will still need to be handled. Fourthly, we did not compare the outcomes of this Mitroflow bioprosthesis (models 12A and LX with no anticalcification treatment) with another bioprosthesis. A comparison of the efficacy of an alternative bioprosthesis was beyond the scope of this study, which focused on methodologic solutions for a precise appraisal of SVD incidence and patient survival after SVD. The results we reported make the case for future randomized clinical trials to evaluate the most effective bioprosthesis. Finally, by exclusively using the Wald test for variable selection, we decided to adopt an explorative approach. There is thus an increase in the first error rate with some possible overfitting issues. Our results need to be confirmed in the future by other studies. We have also completed the evaluation of our model regarding the competing risk analysis with comparisons of results obtained by different methods. As illustrated in the Figure E2, the main conclusion is an underestimation of the CIF by the last 2 methods; that is, by ignoring the interval censoring.

Examination of the literature concerning other bioprostheses reveals that the incidence and definition of SVD are variable, with several large series reporting low rates of SVD in the long term. Bourguignon and colleagues ${ }^{34}$ evaluated 2758 Carpentier Edwards Perimount prostheses (Edwards Lifesciences, Irvine, Calif), with no reported SVD (defined as severe aortic stenosis or severe aortic regurgitation) at 15 years of $78.6 \% \pm 2.2 \%$. Cox proportional hazards regression was used to identify risk factors. For the same bioprosthesis, Forcillo and colleagues $^{35}$ reported freedom from SVD of $34 \% \pm 2 \%$ at 15 years, using the Kaplan-Meier method to estimate the SVD survival curve. Another large series, reported by Mohammadi and colleagues in 2012, ${ }^{36}$ reported freedom from SVD at 15 years for the Freestyle bioprosthesis (Medtronic, Minneapolis, Minn) of $82.3 \%$, using a Cox model to assess the risk factors of SVD. Unfortunately, the last 2 studies mentioned failed to precisely report the SVD criteria they used. Overall, our results are difficult to compare because the previous studies did not consider interval censoring or the competition between death and SVD diagnosis.

The SVD definition we used might be improved. Very recently, Capodanno and colleagues ${ }^{37}$ published some standardized definitions of SVD based on several 
echographic parameters. Close to our own definition, a combined set of criteria for severe hemodynamic SVD is described: mean gradient $>40 \mathrm{~mm} \mathrm{Hg}$, and/or a 20-mm $\mathrm{Hg}$ change from baseline and/or severe aortic regurgitation. To deal with repeated measurements of hemodynamic changes as longitudinal markers, it would have been interesting to use joint modeling, allowing dynamic prediction of the individual risk of death or explant for SVD according to gradient evolution. It considers the variability of the longitudinal marker for a given patient to precisely model its evolution. It should be noted that nonlinear marker evolution can be modeled, as previously reported for the gradient evolution. ${ }^{38}$ Specifically, such joint models have recently been used in patients who received a human tissue valve in the aortic position. ${ }^{39}$ Nevertheless, the main limitation of joint models is that they are based on 1 or 2 longitudinal markers. In our context, valve deterioration can be defined based on several markers (such as mean aortic gradient, aortic valve area, and the presence of aortic regurgitation). We therefore dichotomized echocardiogram-based markers, which is a limitation of our approach. ${ }^{40}$ Introducing these longitudinal measurements in joint models constitutes a future perspective of our work and could represent a solution for developing a dynamic score to help clinicians in their decision to re-operate ${ }^{41}$ and in personalizing screening intervals. ${ }^{39}$ In these models, the baseline of the surgery would be the first postoperative day. At last, although numerous explanatory variables were analyzed, we cannot exclude the possibility that some confounding factors, which were not taken into account in the present study, could have exerted influence.

\section{CONCLUSIONS}

Our study demonstrated an unexpected high incidence of SVD with models 12A and LX of the Mitroflow valves. This issue may have been underestimated due to methodologic pitfalls: the SVD definition based on histologic criteria, the insufficient follow-up period, and the use of traditional statistical approaches for time-to-event data (Kaplan-Meier curves and/or Cox regression) that ignore interval censoring and competition between events. Our results have an immediate influence for patients with a Mitroflow valve. We highly recommend annual follow-ups, especially for patients with SVD risk factors such as severe PPM, dyslipidemia, or COPD. We also hope that our results will encourage clinical trials and other cohort-based studies to describe/compare the outcomes of other bioprotheses. This literature is crucial to avoid underestimation of post-AVR complications.

\section{Conflict of Interest Statement}

Dr Le Tourneau received financial support from Edwards Lifesciences to attend an international meeting. Dr Roussel received consultant fees from Edwards Lifesciences and financial support to attend an international meeting from Edwards Lifesciences, Medtronic, and St. Jude Medical. Dr Sénage received financial support to attend an international meeting from Edwards Lifesciences. All other authors have nothing to disclose with regard to commercial support.

\section{References}

1. Brown JM, O'Brien SM, Wu C, Sikora JA, Griffith BP, Gammie JS Isolated aortic valve replacement in North America comprising 108,687 patients in 10 years: changes in risks, valve types, and outcomes in the Society of Thoracic Surgeons national database. J Thorac Cardiovasc Surg. 2009;137: 82-90.

2. Le Tourneau T, Savoye C, McFadden EP, Grandmougin D, Carton HF, Hennequin JL, et al. Mid-term comparative follow-up after aortic valve replacement with Carpentier-Edwards and pericarbon pericardial prostheses. Circulation. 1999;100(19 Suppl):II11-6.

3. Rahimtoola SH. Choice of prosthetic heart valve in adults: an update. J Am Coll Cardiol. 2010;55:2413-26.

4. Gerosa G, Tarzia V, Rizzoli G, Bottio T. Small aortic annulus: the hydrodynamic performances of 5 commercially available tissue valves. J Thorac Cardiovasc Surg. 2006;131:1058-64.

5. Minami K, Zittermann A, Schulte-Eistrup S, Koertke H, Korfer R. Mitroflow synergy prostheses for aortic valve replacement: 19 years experience with 1,516 patients. Ann Thorac Surg. 2005;80:1699-705.

6. Yankah CA, Pasic M, Musci M, Stein J, Detschades C, Siniawski H, et al. Aortic valve replacement with the Mitroflow pericardial bioprosthesis: durability results up to 21 years. J Thorac Cardiovasc Surg. 2008;136:688-96.

7. Bach DS, Siao D, Girard SE, Duvernoy C, McCallister BD Jr, Gualano SK. Evaluation of patients with severe symptomatic aortic stenosis who do not undergo aortic valve replacement: the potential role of subjectively overestimated operative risk. Circ Cardiovasc Qual Outcomes. 2009;2:533-9.

8. Iung B, Cachier A, Baron G, Messika-Zeitoun D, Delahaye F, Tornos P, et al. Decision-making in elderly patients with severe aortic stenosis: why are so many denied surgery? Eur Heart J. 2005;26:2714-20.

9. Akins CW, Miller DC, Turina MI, Kouchoukos NT, Blackstone EH, Grunkemeier GL, et al. Guidelines for reporting mortality and morbidity after cardiac valve interventions. Eur J Cardiothorac Surg. 2008;33:523-8.

10. Senage T, Le Tourneau T, Foucher Y, Pattier S, Cueff C, Michel M, et al. Early structural valve deterioration of Mitroflow aortic bioprosthesis: mode, incidence, and impact on outcome in a large cohort of patients. Circulation. 2014;130: 2012-20.

11. Leffondre $\mathrm{K}$, Touraine $\mathrm{C}$, Helmer $\mathrm{C}$, Joly P. Interval-censored time-to-event and competing risk with death: is the illness-death model more accurate than the Cox model? Int J Epidemiol. 2013;42:1177-86.

12. Mitroflow aortic pericardial heart valve (model 12) instruction for use. Available at: http://www.accessdata.fda.gov/cdrh_docs/pdf6/P060038c.pdf. Accessed October 1,2018

13. Pibarot P, Dumesnil JG. Hemodynamic and clinical impact of prosthesis-patient mismatch in the aortic valve position and its prevention. J Am Coll Cardiol. 2000; 36:1131-41

14. Akins CW, Miller DC, Turina MI, Kouchoukos NT, Blackstone EH Grunkemeier GL, et al. Guidelines for reporting mortality and morbidity after cardiac valve interventions. J Thorac Cardiovas Surg. 2008;135:732-8.

15. Schemper M, Smith TL. A note on quantifying follow-up in studies of failure time. Control Clin Trial. 1996;17:343-6.

16. Joly P, Commenges D, Helmer C, Letenneur L. A penalized likelihood approach for an illness-death model with interval-censored data: application to age-specific incidence of dementia. Biostatistics. 2002;3:433-43.

17. Frydman H, Szarek M. Nonparametric estimation in a Markov "illness-death" process from interval censored observations with missing intermediate transition status. Biometrics. 2009;65:143-51.

18. Morgan SL, Todd JJ. A diagnostic routine for the detection of consequential heterogeneity of causal effects. Sociologic Methodol. 2008;38:231-81.

19. Aalen S. An empirical transition matrix for non-homogeneous Markov chains based on censored observations. Scand J Stat. 1978;5:141-50.

20. Gillaizeau F, Dantan E, Giral M, Foucher Y. A multistate additive relative survival semi-Markov model. Stat Methods Med Res. 2017;26:1700-11. 
21. Pohar M, Stare J. Relative survival analysis in R. Computer Methods Programs Biomed. 2006;81:272-8.

22. Huebner M, Wolkewitz M, Enriquez-Sarano M, Schumacher M. Competing risks need to be considered in survival analysis models for cardiovascular outcomes. J Thorac Cardiovasc Surg. 2017;153:1427-31.

23. Manji RA, Lee W, Cooper DK. Xenograft bioprosthetic heart valves: past, present and future. Int J Surg. 2015;23(Pt B):280-4.

24. Briand M, Pibarot P, Despres JP, Voisine P, Dumesnil JG, Dagenais F, et al. Metabolic syndrome is associated with faster degeneration of bioprosthetic valves. Circulation. 2006;114(1 Suppl):I512-7.

25. Mahjoub H, Mathieu P, Senechal M, Larose E, Dumesnil J, Despres JP, et al. ApoB/ApoA-I ratio is associated with increased risk of bioprosthetic valve degeneration. J Am Coll Cardiol. 2013;61:752-61.

26. Flameng W, Herregods MC, Vercalsteren M, Herijgers P, Bogaerts K, Meuris B. Prosthesis-patient mismatch predicts structural valve degeneration in bioprosthetic heart valves. Circulation. 2010;121:2123-9.

27. Piccardo A, Blossier JD, Le Guyader A, Orsel I, Sekkal S, Cornu E, et al. Fate of aortic bioprostheses: an 18-year experience. J Thorac Cardiovasc Surg. 2016; 151:754-761 e1.

28. Svensson LG, Adams DH, Bonow RO, Kouchoukos NT, Miller DC, O'Gara PT, et al. Aortic valve and ascending aorta guidelines for management and quality measures. Ann Thorac Surg. 2013;95(6 Suppl):S1-66.

29. Antunes MJ. Degenerated small bioprostheses: still a challenge. J Thorac Cardiovasc Surg. 2018;155:70-1.

30. Inoue Y, Kotani S, Suzuki S. Chimney technique for aortic valve-on-valve replacement. J Thoracic Cardiovasc Surg. 2018;155:68-9.

31. Jamieson WR, Koerfer R, Yankah CA, Zittermann A, Hayden RI, Ling H, et al. Mitroflow aortic pericardial bioprosthesis-clinical performance. Eur J Cardiothoracic Surg. 2009;36:818-24.

32. Yankah CA, Schubel J, Buz S, Siniawski H, Hetzer R. Seventeen-year clinical results of 1,037 Mitroflow pericardial heart valve prostheses in the aortic position. J Heart Valve Dis. 2005;14:172-9.

33. The Italian study on the Mitroflow postoperative results (ISTHMUS): a 20-year, multicentre evaluation of Mitroflow pericardial bioprosthesis. Eur J Cardiothoracic Surg. 2011;39:18-26.
34. Bourguignon T, Bouquiaux-Stablo AL, Candolfi P, Mirza A, Loardi C, May MA, et al. Very long-term outcomes of the Carpentier-Edwards perimount valve in aortic position. Ann Thorac Surg. 2015;99:831-7.

35. Forcillo J, Pellerin M, Perrault LP, Cartier R, Bouchard D, Demers P, et al. Carpentier-Edwards pericardial valve in the aortic position: 25-years experience. Ann Thoracic Surgery. 2013:96:486-93.

36. Mohammadi S, Tchana-Sato V, Kalavrouziotis D, Voisine P, Doyle D, Baillot R, et al. Long-term clinical and echocardiographic follow-up of the Freestyle stentless aortic bioprosthesis. Circulation. 2012;126(11 Suppl 1):S198-204.

37. Capodanno D, Petronio AS, Prendergast B, Eltchaninoff H, Vahanian A, Modine T, et al. Standardized definitions of structural deterioration and valve failure in assessing long-term durability of transcatheter and surgical aortic bioprosthetic valves: a consensus statement from the European Association of Percutaneous Cardiovascular Interventions (EAPCI) endorsed by the European Society of Cardiology (ESC) and the European Association for Cardio-Thoracic Surgery (EACTS). Eur J Cardiothoracic Surg. 2017;52: 408-17.

38. Johnston DR, Soltesz EG, Vakil N, Rajeswaran J, Roselli EE, Sabik JF III, et al. Long-term durability of bioprosthetic aortic valves: implications from 12,569 implants. Ann Thorac Surg. 2015;99:1239-47.

39. Rizopoulos D, Taylor JM, Van Rosmalen J, Steyerberg EW, Takkenberg JJ. Personalized screening intervals for biomarkers using joint models for longitudinal and survival data. Biostatistics. 2016;17:149-64.

40. Douglas PS, Leon MB, Mack MJ, Svensson LG, Webb JG, Hahn RT, et al. Longitudinal hemodynamics of transcatheter and surgical aortic valves in the PARTNER Trial. JAMA Cardiol. 2017;2:1197-206.

41. Andrinopoulou ER, Rizopoulos D, Takkenberg JJ, Lesaffre E. Joint modeling of two longitudinal outcomes and competing risk data. Stat Med. 2014;33: $3167-78$

Key Words: biologic aortic valve replacement, structural valve deterioration, illness-death model, patient survival 


\section{APPENDIX 1. DESCRIPTION OF THE SEMI-MARKOV (SM) MODEL.}

Let $T$ be the chronological time from baseline and $S$ the duration (or sojourn time) in a state. Let $y$ be the set of possible clinical states. The stochastic process under consideration is $\left\{Y_{m}, T_{m}, m \in \mathbb{N}\right\}$, where $Y_{m}$ is the state of the patient after the m-th transition occurring at time $T_{m}$ with $T_{0}<T_{1} \ldots<T_{m}\left(T_{0}=0\right.$ and $Y_{0}=1$ by convention $)$. Let $\varepsilon$ be the set of possible transitions $i j$ with $(i, j) \in(y, y)$, where $i$ represents a transient state with $j$ distinct from $i$. We use $\delta_{i j}$ to denote the indicator function of a transition ij: $\delta_{i j}=1$ if the transition $i j$ is observed (with the duration time $s_{i j}$ in state $i$ before transition to state $j$ ), and $\delta_{i j}=0$ otherwise. Let $X$ be the vector of patient characteristics at baseline, and $X_{i j}$ the subvector of characteristics specifically associated to the transition $i j$. In the context of the illness-death model, $y=\{1,2,3\}$ with $\{Y=1\}$ the healthy stage, $\{Y=2\}$ the illness stage, and $\{Y=3\}$ the death.

The SM model considers that the transition intensities between 2 states depend on the time already spent in the current state. The hazard function for transition from state $Y_{m}=i$ to the state $Y_{m+1}=j$ after a duration s, given patient characteristics $X_{i j}=x_{i j}$ is therefore defined by:

$$
\begin{aligned}
\lambda_{i j}\left(s \mid x_{i j}\right) & =\lim _{\Delta s \rightarrow 0^{+}} P\left(s \leq T_{m+1}-T_{m}<s+\Delta s, Y_{m+1}\right. \\
& \left.=j \mid T_{m+1}-T_{m}>s, Y_{m}=i, x_{i j}\right) / \Delta s
\end{aligned}
$$

The probability for a patient to spend at least some time $s$ in state $i$, given its characteristics $X_{i}=x_{i}$ at baseline, can be defined as:

$$
S_{i}\left(s \mid x_{i}\right)=\exp \left(-\sum_{j: i j \in \in} \int_{0}^{s} \lambda_{i j}\left(u \mid x_{i j}\right) d u\right)
$$

The corresponding density function specific to transition $i j$ after duration $s$ can be deduced from the previous equations: $f_{i j}\left(s \mid x_{i}\right)=\lambda_{i j}\left(s \mid x_{i j}\right) S_{i}\left(s \mid x_{i}\right)$. Consider a parametric SM model, parameters being estimated by maximizing the log-likelihood. Take a sample of $N$ independent subjects $(h=1, \ldots, N)$. Let $l_{h}$ be the individual contribution to the likelihood for a subject $h$.

In the illness-death context where time-to-illness is interval-censored, 5 observed trajectories are possible (Figure E4).

Trajectory I. At the time of right-censoring $t_{\max }$, the individual $h$ was alive. The illness was never diagnosed, the patient being illness-free at the time $t_{i n f}$, which corresponds to the last visit with a negative diagnosis. In this situation, the illness may have occurred in-between $t_{i n f}$ and $t_{\max }$ or the patient can also be illness-free at $t_{\max }$. The probability of observing this trajectory is:

$$
l_{h}=S_{1}\left(t_{\max } \mid x_{1}\right)+\int_{t_{\text {inf }}}^{t_{\max }} f_{12}\left(u \mid x_{1}\right) S_{2}\left(t_{\max }-u \mid x_{23}, u\right) d u
$$

Trajectory II. At the time of right-censoring $t_{\max }$, the individual $h$ was alive. The illness was diagnosed at the time $t_{\text {sup }}$. The patient was illness-free at the time $t_{\text {inf }}$, which corresponds to the last visit with a negative diagnosis. The illness have occurred in-between $t_{\text {inf }}$ and $t_{\text {sup }}$. The probability of observing this trajectory is:

$$
l_{h}=\int_{t_{\text {inf }}}^{t_{\text {sup }}} f_{12}\left(u \mid x_{1}\right) S_{2}\left(t_{\max }-u \mid x_{23}, u\right) d u
$$

Trajectory III. The individual $\mathrm{h}$ died at the time $t_{\max }$. The illness was diagnosed at the time $t_{\text {sup }}$. The patient was illness-free at $t_{i n f}$, which corresponds to the last visit with a negative diagnosis. The illness have occurred in-between $t_{\text {inf }}$ and $t_{\text {sup }}$. The probability of observing this trajectory is:

$$
l_{h}=\int_{t_{\text {inf }}}^{t_{\text {sup }}} f_{12}\left(u \mid x_{1}\right) f_{23}\left(t_{\max }-u \mid x_{23}, u\right) d u
$$

Trajectory IV. The individual $h$ died at the time $t_{\max }$. The illness was never diagnosed, the patient being illness-free at the time $t_{i n f}$, which corresponds to the last visit with a negative diagnosis. The illness may have occurred in-between $t_{i n f}$ and $t_{\max }$ or the patient may also die without structural valve deterioration. The probability of observing this trajectory is:

$$
l_{h}=f_{13}\left(t_{\max } \mid x_{1}\right)+\int_{t_{\text {inf }}}^{t_{\max }} f_{12}\left(u \mid x_{1}\right) f_{23}\left(t_{\max }-u \mid x_{23}, u\right) d u
$$

In the multivariable SM model and under the proportional hazard assumption, the hazard function for transition from state $Y_{m}=i$ to the state $Y_{m+1}=j$ after a durations, given patient characteristics $X_{i j}=x_{i j}$ was defined as follows:

$$
\lambda_{i j}\left(s \mid x_{i j}\right)=\lambda_{0, i, j}(s) \exp \left(\beta_{i j} x_{i j}\right)
$$

where $\lambda_{0, i, j}(s)$ is the baseline hazard function when all the covariates equal 0 and $\beta_{i j}$ are the regression coefficients for transition from state $Y_{m}=i$ to the state $Y_{m+1}=j$. The interpretation of one regression coefficient is possible given that all the other covariates are fixed. The hazard ratio equals the exponential of the regression coefficient. 
Parameters of the SM model are estimated by maximizing the $\log$-likelihood; that is, $\Sigma_{h=1}^{N} \log l_{h}$. We used R statistical software version 3.0.1 ( $\mathrm{R}$ Foundation for Statistical Computing, Vienna, Austria) with optim() to maximize the log-likelihood function of the SM model and to compute the corresponding Hessian matrix (Nelder and Mead algorithms). The complete methodology has been implemented in the multistate R package (available at www.labcom-risca. com) or on the Comprehensive R Archive Network (available at www.cran.r-project.org) mirrors. 


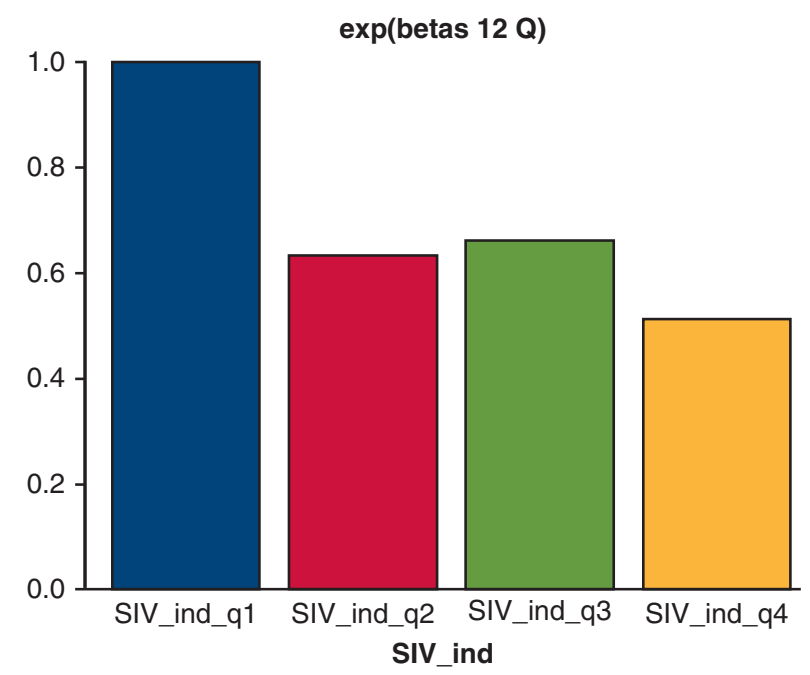

FIGURE E1. Beta exponential for the quartiles of the indexed in vivo surface area, regarding the transition between state 1 (healthy with an aortic valve replacement) and state 2 (proven structural valve deterioration). SIV_ind_q2 is the beta exponential for the 2nd quartile of the indexed in vivo surface area. First quartile served as the reference. SIV, Indexed in vivo surface area.

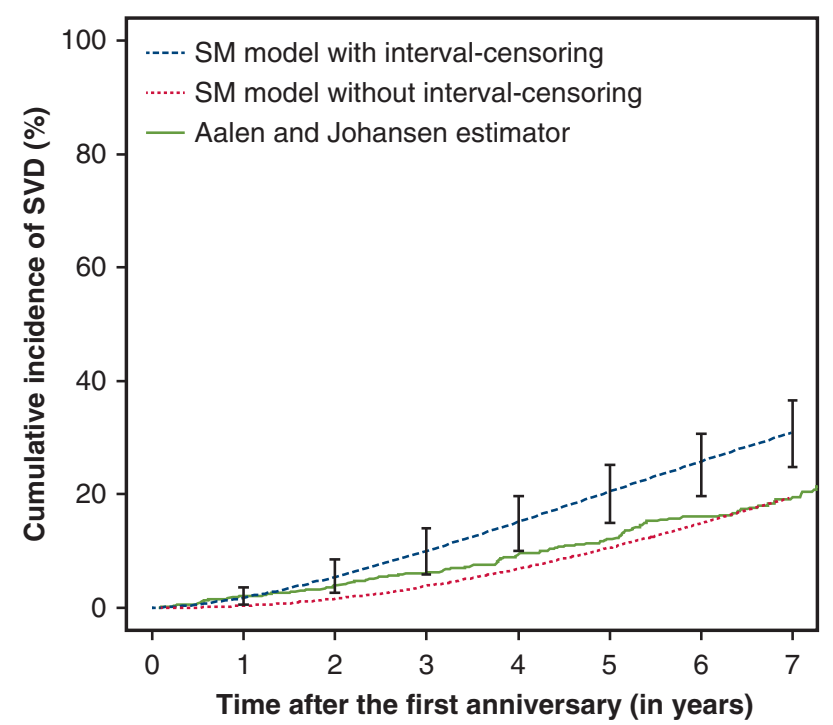

FIGURE E2. Comparison of the cumulative incidence function of structural valve deterioration (SVD), obtained by the proposed semi-Markov ( $S M$ ) illness-death model, the same model without taking into account interval-censoring, and the Aalen and Johansen estimator.

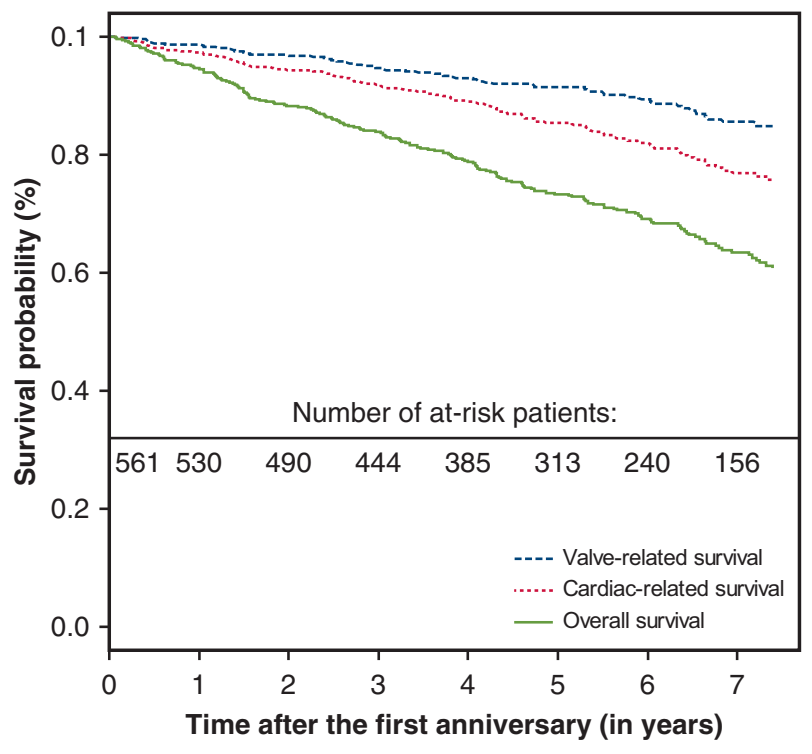

FIGURE E3. Overall valve-related and cardiac-related patient survival. 


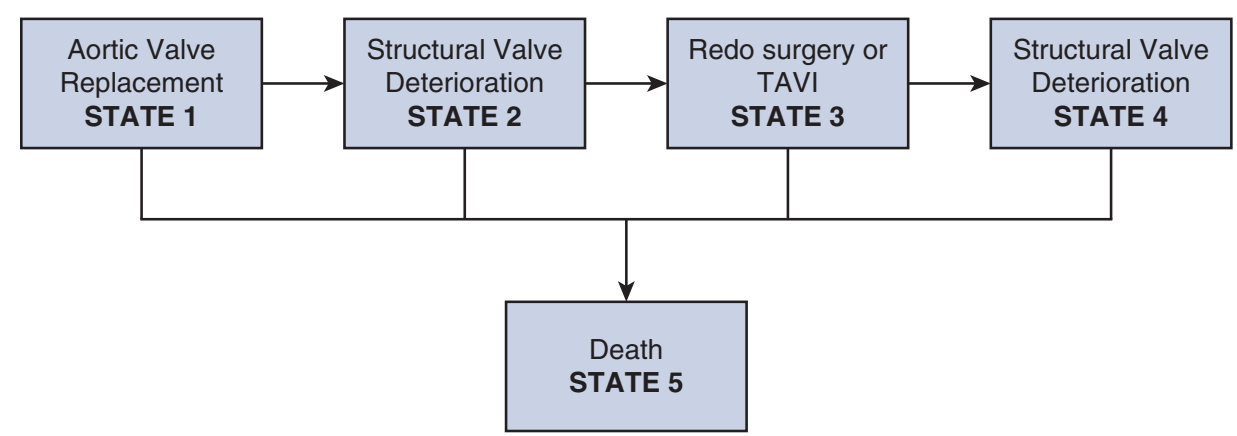

FIGURE E4. Optimal multistate model to represent the detailed evolution for a patient following an aortic valve replacement (AVR). TAVI, Transcatheter aortic valve implantation.

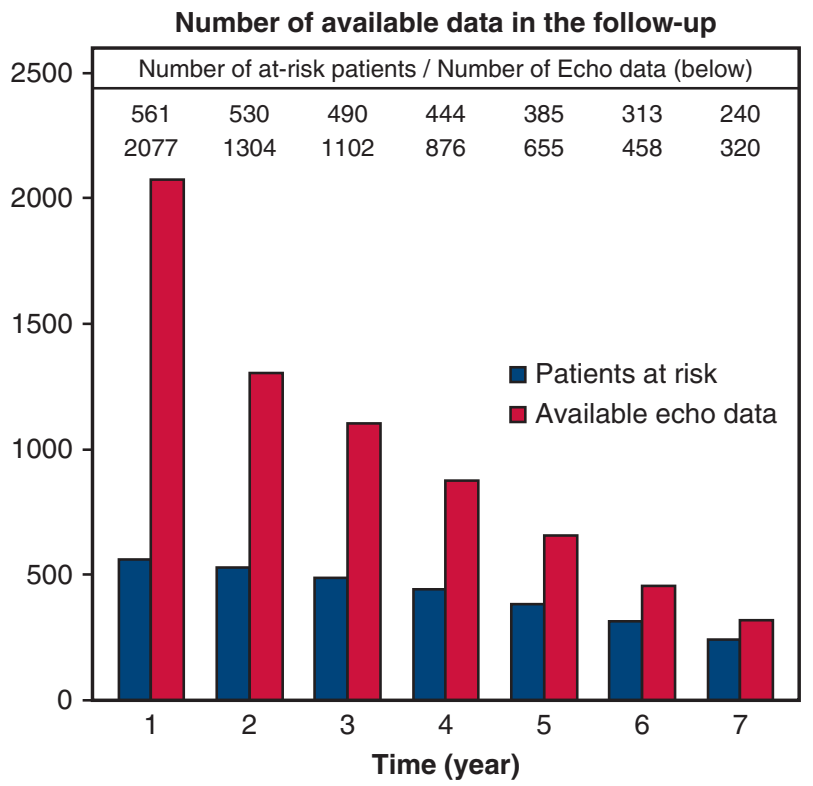

FIGURE E5. Number of available data according to the time of follow-up.

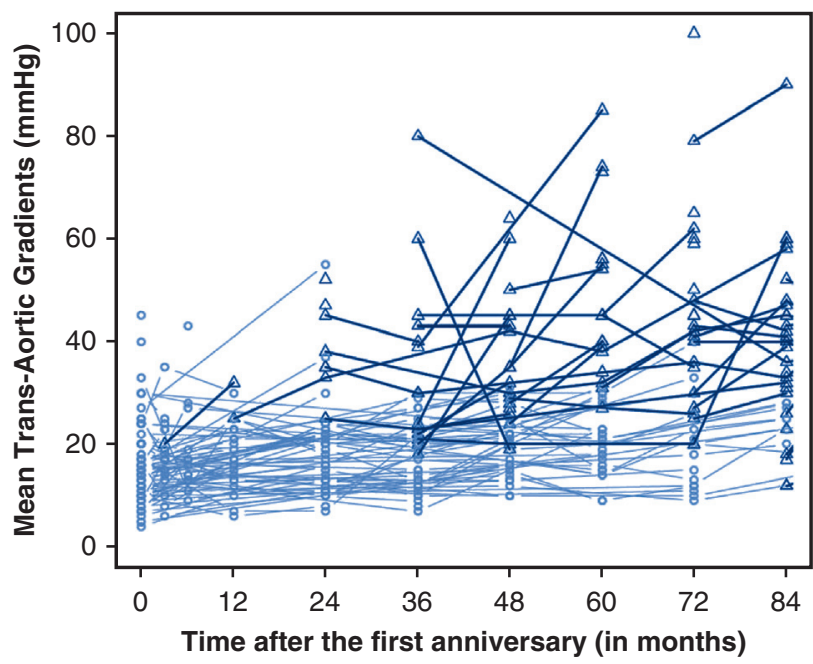

FIGURE E6. Mean gradient evolution for the 103 cases of structural valve deterioration (SVD). Line is in grey before the SVD diagnosis time, and blue following the SVD diagnosis. 




FIGURE E7. Cumulative incidence of structural valve deterioration $(S V D)$ after the first anniversary of surgery. The red curve was estimated from the illness-death model for the 561 patients. The blue curve was estimated from the same model without the 9 patients missing echocardiogram data.
TABLE E2. Significant factors associated with the time-to-structural valve deterioration after the first anniversary of surgery from the illness-death model (forced age adjustment), for the 561 on the left and for the $\mathbf{5 5 2}$ without the 9 patients missing echocardiogram data on the right

\begin{tabular}{|c|c|c|}
\hline & $\begin{array}{c}\text { Hazard ratio } \\
(95 \% \text { confidence } \\
\text { interval) } \\
(\mathbf{n}=\mathbf{5 6 1})\end{array}$ & $\begin{array}{c}\text { Hazard ratio } \\
\text { (95\% confidence } \\
\text { interval) } \\
(\mathbf{n}=\mathbf{5 5 2})\end{array}$ \\
\hline $\begin{array}{l}\text { Severe patient-prosthesis } \\
\text { mismatch }\end{array}$ & $1.68(1.13-2.49)$ & $1.70(1.14-2.51)$ \\
\hline Female & $1.59(1.06-2.38)$ & $1.61(1.08-2.43)$ \\
\hline Dyslipidemia & $1.56(1.07-2.27)$ & $1.10(1.59-2.31)$ \\
\hline $\begin{array}{l}\text { Chronic obstructive } \\
\text { pulmonary disease }\end{array}$ & $2.91(1.56-5.44)$ & $2.99(1.61-5.54)$ \\
\hline $\begin{array}{l}\text { Younger than age } 70 \mathrm{y} \\
\text { at the time of surgery }\end{array}$ & 1.00 & 1.00 \\
\hline $\begin{array}{l}\text { Between ages } 70 \text { and } 80 y \\
\text { at time of surgery }\end{array}$ & $1.05(0.62-1.78)$ & $1.01(0.59-1.72)$ \\
\hline $\begin{array}{l}\text { Older than age } 80 \mathrm{y} \text { at time } \\
\text { of surgery }\end{array}$ & $0.90(0.47-1.72)$ & $0.86(0.58-1.28)$ \\
\hline
\end{tabular}

TABLE E1. Causes of death for the 190 observed deaths

\begin{tabular}{lrr}
\hline \multicolumn{1}{c}{ Cause of death } & n & $\%$ \\
\hline Congestive heart failure & 34 & 17.9 \\
\hline Cancer & 24 & 12.6 \\
\hline Structural valve deterioration & 19 & 10.0 \\
\hline Sepsis & 18 & 9.5 \\
\hline Sudden death & 11 & 5.8 \\
\hline Respiratory failure & 8 & 4.2 \\
\hline Cerebral vascular accident & 7 & 3.7 \\
\hline Endocarditis & 6 & 3.2 \\
\hline Myocardial infarction & 6 & 3.2 \\
\hline Multiorgan failure & 3 & 1.6 \\
\hline Thromboembolic event & 3 & 1.6 \\
\hline Digestive bleeding & 2 & 1.1 \\
\hline Cardiogenic shock & 2 & 1.1 \\
\hline Mesenteric ischemia & 2 & 1.1 \\
\hline Other & 20 & 10.5 \\
\hline Unknown & 25 & 13.2 \\
\hline Total & 190 & 100.0 \\
\hline
\end{tabular}

